\title{
Serological Gharacters of the Pediococci
}

\author{
By HELGE L. GÜNTHER AND HELEN R. WHITE \\ Bacteriology Department, Queen Elizabeth College (University of London), \\ London, W. 8
}

(Received 17 November 1960)

SUMMARY

Rabbit antisera were prepared against 12 pediococcus isolates and tested by precipitin, precipitin-absorption and agglutination tests. Common precipitins for the three species Pediococcus cerevisiae, $\boldsymbol{P}$. parvulus n.sp. and $\boldsymbol{P}$. damnosus were demonstrated. An antiserum prepared against Aerococcus viridans (NCTC 7599) reacted with extracts of the homologous strain and 7 out of 10 heterologous Aerococcus viridans.

\section{INTRODUCTION}

Renewed interest in the genus Pediococcus has led in recent years to extensive studies of the cultural and physiological characters of its members (Pederson, 1949; Felton \& Niven, 1953; Pederson, Albury \& Breed, 1954; Jensen \& Seeley, 1954) but, so far, no study has been reported of the serological properties of the organisms. In view of the successful application of precipitin and agglutination tests in the characterization of other lactic acid bacteria it was decided to explore the possibility of using serological methods in the classification of pediococci. Some preliminary results of this investigation are reported in this paper.

\section{METHODS}

The cultures and methods of cultivation are described in our previous paper (Günther \& White, 1961).

Organisms. Twelve strains representative of the physiological groups described in the same previous publication were selected for antisera production.

Pediococcus cerevisiae (group I) was represented by strains 8081, F-166, 2-170, N-91, A-1, FP-1; P. parvulus (group II) by strains M-31, A-181, S-190, SS-69; $P$. damnosus (possible group IV) by strain M-1, and $P$. halophilus (possible group V) by strain Tc. 1 .

We intended to prepare an antiserum for strain L-148, which belongs to the unnamed group (group III), but this proved unsatisfactory and no further attempts have as yet been made to prepare antisera against strains belonging to this group.

One antiserum against Aerococcus viridans H-9 (NCTC 7599) was prepared.

Preparation of antisera. Intravenous injections of $(a)$ whole living organisms, or (b) disintegrated suspensions, were given to rabbits which had been tested previously for the absence of naturally occurring antibodies. For method (a) $40 \mathrm{ml}$. glucose Lemco (GL) broth (Shattock \& Hirsch, 1947) were inoculated with $0.5 \mathrm{ml}$. of a $24 \mathrm{hr}$. 'Oxoid' tomato juice (TJ) broth culture and incubated for $24 \mathrm{hr}$. at $30^{\circ}$. The culture was centrifuged, washed twice in $0.9 \%(\mathrm{w} / \mathrm{v})$ sodium chloride solution 
resuspended in $0.9 \%$ sodium chloride solution to give an opacity equivalent to Brown's tube No. 7 and then stored at $4^{\circ}$ for use in one course of animal injections. In method $(b)$ fresh suspensions of organisms were prepared for each injection. Organisms from $20 \mathrm{ml}$. of a culture in GL broth were washed and adjusted to the same optical density as in method (a). They were then disrupted for $30 \mathrm{~min}$. at room temperature in a tissue disintegrator (Mickle, 1948) in a vessel containing $5 \mathrm{~g}$. sterile Ballotini beads grade No. 12. After centrifugation the supernatant fluid was found to be sterile and was used for animal injection.

When the two methods of antibody production were compared it was noted that the disintegration of the organism before inoculation did not result in a speedier production of antibodies, nor was the potency of the resulting antiserum increased. Suspensions of whole living organisms were therefore used for the routine preparation of antisera; the results recorded below refer in all instances to sera prepared by this method.

The experimental animals received $1 \mathrm{ml}$. volumes of suspensions of organisms prepared as above at 3-4 days intervals. One course of injections consisted of five such inoculations. When a test bleeding at this stage indicated that the antibody produced was of insufficient potency, a second course was given. Occasionally, a third course had to be administered before satisfactory antibody was obtained. The animals were bled 3 or 4 days after the last injection. Sera were stored at $4^{\circ}$ without preservative.

The presence of non-specific antibodies was excluded by precipitin tests with each serum against extracts of streptococci (representative of Lancefield's groups A to N) and against extracts of 7 staphylococcus and 3 leuconostoc strains.

Preparation of extracts. (a) Crude extracts: Lancefield's (1933) $\mathrm{HCl}$ extraction method was used as modified by Sharpe (1955). The cultures were incubated for $48 \mathrm{hr}$. at $30^{\circ}$ in tomato juice broth. The reactions of at least two extracts, prepared at different times, were tested to ensure reproducibility of results. (b) Ethanol precipitation. The difficulty in reading the results with some of the sera and crude. extracts prepared as above made it desirable to concentrate the antigenically active material in the extracts by ethanol precipitation as suggested by Shattock (1949) for group D streptococci. A slight increase in activity in the precipitate was noted with most of the extracts, but in a few this treatment resulted in the complete loss of activity in the precipitate as well as in the supernatant fluid. Ethanol precipitation was not used, therefore, for the routine preparation of extracts.

Precipitin tests. Ring tests were carried out as described by Sharpe (1955). Observations were continued for $30 \mathrm{~min}$., or up to $60 \mathrm{~min}$. when a loss of potency in the antiserum was noted after prolonged storage. Results obtained with antisera which had been stored for a long time were always regarded as unreliable but were useful in preliminary work.

Precipitin-absorption tests. The organisms were cultivated in tomato juice broth for $24 \mathrm{hr}$. at $30^{\circ}$. The technique outlined by Sharpe (1955) was followed.

Agglutination tests. Tube agglutination, employing suspensions of organisms from $16 \mathrm{hr}$. tomato juice broth cultures in $0.9 \%(\mathrm{w} / \mathrm{v})$ sodium chloride solution, was used. The tubes were read after overnight incubation at $50^{\circ}$. 


\section{RESULTS}

The selected pediococcus isolates differed in their capacity to induce antibody response in the rabbit and the choice of suitable isolates for antiserum production was of great importance. The difficulty experienced in obtaining active extracts with some isolates merits further investigation. In the future, modifications may

Table 1. Precipitin tests with antisera of pediococci and Aerococcus viridans Antisera

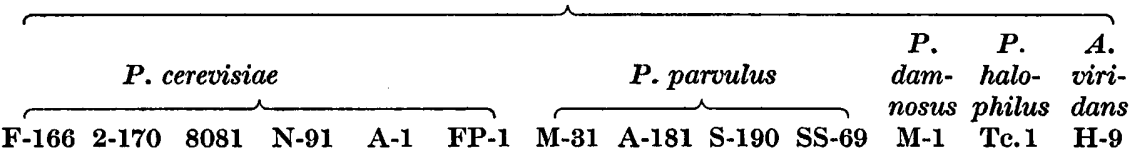

P. cerevisiae (group I)

\begin{tabular}{|c|c|c|c|c|c|c|c|c|c|c|c|c|c|}
\hline F-166 & $+t$ & $t$ & + & + & + & + & + & + & + & + & \pm & - & - \\
\hline 2-170 & + & ++ & + & \pm & \pm & + & + & + & + & \pm & - & - & - \\
\hline 8081 & + & + & ++ & + & + & + & + & + & + & + & + & - & - \\
\hline N-91 & + & + & + & ++ & + & + & + & \pm & - & + & - & - & - \\
\hline A-1 & + & + & + & - & ++ & + & - & - & - & - & - & - & - \\
\hline FP-1 & + & + & + & - & - & ++ & + & - & - & + & - & - & - \\
\hline Others & $+\frac{30}{31}$ & $+\frac{29}{31}$ & $+\frac{27}{31}$ & $+\frac{24}{30}$ & $+\frac{26}{30}$ & $+\frac{29}{31}$ & $+\frac{24}{30}$ & $+\frac{14}{16}$ & $+\frac{18}{30}$ & $+\frac{28}{30}$ & $+\frac{21}{28}$ & $-\frac{28}{28}$ & $-\frac{\mathbf{3 0}}{\mathbf{3 0}}$ \\
\hline
\end{tabular}

$P$. parvulus (group II)

M-3I

A-181

S-190

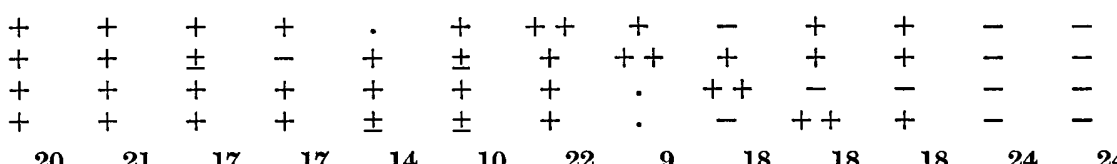

Others

$+\frac{20}{24}+\frac{21}{24}-\frac{17}{24}-\frac{17}{24}-\frac{14}{18}+\frac{10}{24}+\frac{22}{24}-\frac{9}{13}-\frac{18}{23}+\frac{18}{24}+\frac{18}{24}-\frac{24}{24}-\frac{24}{24}$

P. damnosus

(? group IV)

M-1

Others

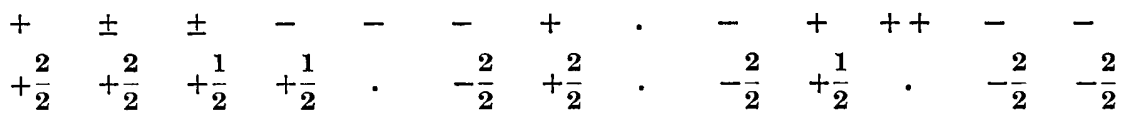

P. halophilus Tc. 1

(? group V)

\section{A. viridans}

H-9

Others

Leuconostocs

Streptococci

Staphylococci

Staph. aureus 7447

Staph. albus 7292

Others

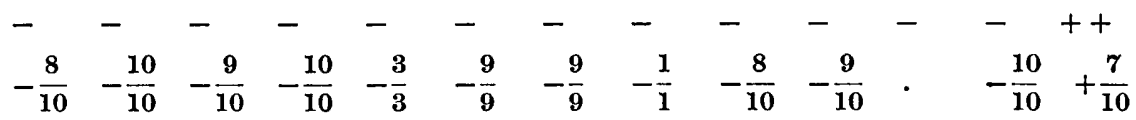$$
\begin{array}{llllllll}
-\frac{3}{3} & -\frac{2}{3} & -\frac{3}{3} & . & -\frac{3}{3} & -\frac{3}{3} & . & -\frac{3}{3}
\end{array}
$$$$
-\frac{24}{24}-\frac{24}{24}-\frac{24}{24} \quad-\frac{12}{24} \quad-\frac{12}{24} \quad-\frac{24}{24} \quad-\frac{12}{24} \quad-\frac{24}{24} \quad-\frac{12}{24}-\frac{12}{24}-\frac{12}{24} \quad-\frac{12}{24} \quad-\frac{24}{24}
$$ 
have to be made in the method of preparation in order to obtain satisfactory extracts of consistently high activity.

Precipitin tests. These results are summarized in Table 1. Positive reactions were obtained with extracts of the homologous and with the majority of heterologous strains of the same species. Antisera against Pediococcus cerevisiae strains F-166, 2-170, and FP-1 also precipitated consistently with extracts of $P$. parvulus. Antisera against $\boldsymbol{P}$. cerevisiae strains $8081, \mathbf{N}-91$, and A-1 were less consistent in their reactions with extracts of $\boldsymbol{P}$. parvulus, but they too gave positive reactions with a large number of extracts of that species.

Antisera against $\boldsymbol{P}$ ediococcus parvulus and $\boldsymbol{P}$. damnosus reacted with the homologous and with the majority of the heterologous extracts of the same species as well as with extracts of $\boldsymbol{P}$. cerevisiae. Cross-reactions occurred also between $\boldsymbol{P}$. parvulus and $\boldsymbol{P}$. damnosus.

Reactions could not be observed between sera against Pediococcus cerevisiae, $\boldsymbol{P}$. parvulus or $\boldsymbol{P}$. damnosus on the one hand, and between 'group III' isolates or $\boldsymbol{P}$. halophilus on the other. The serum against $\boldsymbol{P}$. halophilus was not precipitated by extracts of any of the other pediococci.

Table 2. Absorption of antisera of Pediococcus cerevisiae strains $F-166$ and 2-170 with cells of homologous and heterologous $\mathrm{P}$. cerevisiae strains

\begin{tabular}{|c|c|c|c|c|c|c|c|c|c|c|}
\hline \multirow[b]{4}{*}{$\begin{array}{c}\text { Antigen } \\
\text { (extracts) }\end{array}$} & \multicolumn{9}{|c|}{ Antiserum } & \\
\hline & \multicolumn{4}{|c|}{ F-166 } & \multicolumn{6}{|c|}{$2-170$} \\
\hline & \multirow[b]{2}{*}{$\begin{array}{l}\text { Unab- } \\
\text { sorbed }\end{array}$} & \multicolumn{3}{|c|}{ Absorbed with strains } & \multirow[b]{2}{*}{$\begin{array}{l}\text { Unab- } \\
\text { sorbed }\end{array}$} & \multicolumn{5}{|c|}{ Absorbed with strains } \\
\hline & & F-166 & $\overbrace{\mathrm{D}-32}$ & $\widehat{\text { PUE }}$ & & 2-170 & F-166 & 8081 & FP-1 & A-1 \\
\hline \multicolumn{11}{|c|}{ P. cerevisiae (group I) } \\
\hline F-166 & $+t$ & - & - & + & + & - & - & - & - & - \\
\hline $2-170$ & + & - & - & - & ++ & - & 一 & . & . & . \\
\hline PUE & + & - & . & - & + & . & . & . & . & . \\
\hline Others & + & - & - & - & + & - & - & - & - & - \\
\hline $\begin{array}{c}\text { P. parvulus } \\
\text { (group II) }\end{array}$ & + & - & - & - & + & . & . & - & - & - \\
\hline
\end{tabular}

Symbols as Table 1.

Six of the pediococcus antisera gave positive reactions with one or the other aerococcus extract, but in these instances ring formation was markedly delayed and considerably less intense than that observed with the pediococci.

The Aerococcus viridans H-9 serum reacted with extracts of the homologous and with 7 of the 10 other strains of $A$. viridans tested. A weak cross-reaction was obtained also with extracts of Pediococcus halophilus and with strain L-171 of the unclassified group III.

Cross-reactions were not found between the pediococcus antisera used and the extracts of streptococci, staphylococci and leuconostoc strains, with the exception of one reaction between a strain of Staphylococcus albus and Pediococcus halophilus antiserum.

Precipitin-absorption tests. Four antisera were selected for absorption tests. The results are shown in Tables 2, 3, 4. These results indicate that certain antisera contained more than one antibody, one of which was absorbed by heterologous 
Table 3. Absorption of antiserum Pediococcus cerevisiae strain F-166 with cells of P. parvulus and strain L-148 (group III)

\begin{tabular}{|c|c|c|c|c|c|c|}
\hline \multirow[b]{2}{*}{$\begin{array}{l}\text { Antigen } \\
\text { P. cerevisiae (group I) }\end{array}$} & \multicolumn{6}{|c|}{ Antiserum F-166 absorbed with strains $P$. parvulus } \\
\hline & $\begin{array}{c}\text { Un- } \\
\text { absorbed }\end{array}$ & A-181 & S-190 & SS-69 & S-182 & L-148 \\
\hline F-166 & ++ & + & - & + & - & + \\
\hline $2-170$ & + & - & - & - & - & + \\
\hline PUE & + & . & - & - & - & - \\
\hline Others & + & - & - & - & - & - \\
\hline P. parvulus (group II) & + & - & - & - & - & + \\
\hline
\end{tabular}

Table 4. Absorption of antisera Pediococcus parvulus strains $S-190$ and SS-69 with cells of $\mathbf{P}$. cerevisiae and $\mathbf{P}$. parvulus

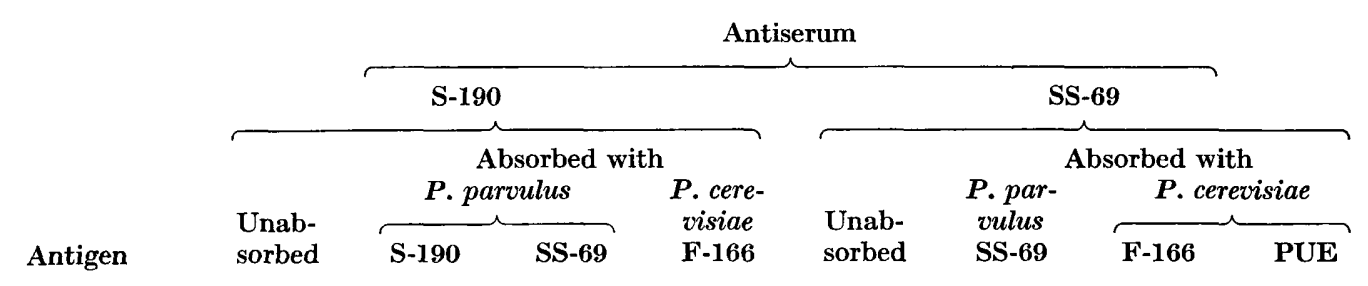

P. parvulus (group II)

S-190

SS-69

Others

P. cerevisiae

(group I)

$\begin{array}{llllllll}+ & - & + & + & + & - & - & - \\ + & - & + & - & + & - & + & + \\ + & - & - & - & + & - & - & - \\ + & - & - & - & + & - & - & -\end{array}$

Symbols as Table 1 .

Table 5. Agglutination tests with pediococcus and aerococcus antisera

\begin{tabular}{|c|c|c|c|c|c|c|c|c|}
\hline \multirow[b]{3}{*}{ Antigen } & \multicolumn{8}{|c|}{ Antisera } \\
\hline & \multicolumn{4}{|c|}{ P. cerevisiae } & & \multicolumn{2}{|c|}{ P. parvulus } & \multirow{2}{*}{$\begin{array}{c}\text { A. viri- } \\
\text { dans } \\
\text { H-9 }\end{array}$} \\
\hline & F-166 & 2-170 & 8081 & N-91 & FP-1 & $M-31$ & SS-69 & \\
\hline \multicolumn{9}{|c|}{ cerevisiae (group I) } \\
\hline F-166 & $1280+$ & - & - & - & - & - & - & - \\
\hline 2-170 & 80 & $1280+$ & - & 40 & 80 & - & - & - \\
\hline 8081 & 20 & 40 & $1280+$ & 40 & 40 & 40 & 40 & 20 \\
\hline N-91 & - & - & - & 640 & - & - & - & - \\
\hline FP-1 & 40 & 80 & - & 40 & $1280+$ & - & 160 & - \\
\hline \multicolumn{9}{|c|}{ parvulus (group II) } \\
\hline M-31 & - & - & - & - & - & $1280+$ & - & - \\
\hline SS-69 & 320 & 40 & 40 & 40 & 160 & 80 & 1280 & 40 \\
\hline $\begin{array}{l}\text { viridans } \\
\text { H-9 }\end{array}$ & - & - & - & - & - & - & 320 & $1280+$ \\
\hline
\end{tabular}

Figures are the reciprocals of the highest titres read. $-=>20$. 
strains and the other absorbed only by the homologous strain. Antibodies in Pediococcus cerevisiae antiserum were absorbed by $P$. parvulus strains and vice versa, indicating that these two species share at least one common antigen. Absorption with strain L-148 organisms, with strains of Aerococcus viridans, streptococci and staphylococci did not result in loss of antibodies from the antisera of $P$. cerevisiae or $\boldsymbol{P}$. parvulus.

Agglutination tests. The results are shown in Table 5. With homologous strains titres of more than 1/1280 were obtained generally but with heterologous strains, when any reaction at all was obtained, the titres seldom exceeded 1/80. A number of cross-reactions occurred between antisera of Pediococcus cerevisiae and $P$. parvulus. In one instance cross-reactions occurred between $P$. parvulus strain SS-69 and Aerococcus viridans strain $\mathbf{H}-9$.

\section{DISCUSSION}

The present investigation indicates that serological techniques may be applied successfully to the separation and classification of the pediococci. Antisera prepared against pediococci did not react with extracts of organisms from the closely related genera Streptococcus and Leuconostoc and generally not with extracts from aerococci. It becomes evident that pediococci possess precipitins which are common to members of more than one species. Such antigens were demonstrated in Pediococcus cerevisiae, $\boldsymbol{P}$. parvulus and $\boldsymbol{P}$. damnosus, but not in $\boldsymbol{P}$. halophilus and in members of the as yet unclassified subgroup (III). As these antigens were shared by more than one species they may be referred to as 'group' antigens by analogy to the group antigens of streprococci.

Some pediococcus strains showed an additional antigen which appeared to be more strain specific and may therefore represent a 'type' antigen. The presence of more than one antigen was also demonstrated in agglutination tests. These tests revealed relationships apparently much narrower than were demonstrated in precipitin tests. It is probable, then, that pediococci possess a 'group' antigen demonstrable by precipitin tests and a 'type' antigen which can be shown by precipitin and agglutination tests, as with streptococci and lactobacilli. No study has as yet been made to determine whether the 'type' antigens demonstrable in the two tests were identical. The possibility that the organisms possess more than one 'type' antigen cannot be excluded.

Some indication that the 'group' antigen may be protein in nature was obtained from ethanol precipitation of some of the extracts since the antigenically active material had been found in the precipitate. However, the antigen might be carbohydrate carried down with the protein.

The serological position of Pediococcus halophilus and of the unnamed group III could not be established satisfactorily. Absorption of $\boldsymbol{P}$. cerevisiae antiserum with one strain of group III did not remove the 'group' antigen from the serum. This indicates that members of group III are not related antigenically to $\boldsymbol{P}$. cerevisiae (group I) and $\boldsymbol{P}$. parvulus (group II). They may possibly represent a separate serological group.

The reaction of strain PUE is of interest. Pederson et al. (1954) excluded it from the pediococci but the strain was classified by us (Günther \& White, 1961) as Pediococcus cerevisiae. This organism gave strong precipitin reactions with antisera 
of $\boldsymbol{P}$. cerevisiae, $\boldsymbol{P}$. parvulus and $\boldsymbol{P}$. damnosus. When PUE was used for absorption of $\boldsymbol{P}$. cerevisiae antiserum the 'group' antigen was absorbed. This is further evidence that the correct classification of this strain is with $\boldsymbol{P}$. cerevisiae.

The serological work also confirms the classification of strain A-2 isolated by Dacre and classified by him tentatively as $\boldsymbol{P}$. cerevisiae (Dacre, 1958) and of the old 'Leuconostoc mesenteroides $\mathrm{P}-60$ ' reclassified by Garvie (1959) as $P$. cerevisiae.

The cross-reactions between the aerococci and pediococci might have been due to non-specific antibodies, but more experimental evidence is required. The work is as yet incomplete but it is being continued and we hope to clarify at least some of the issues raised in this and the preceding paper.

We wish to express our gratitude to all persons who supported this work by providing us with numerous cultures. We are particularly grateful to Dr M. Elizabeth Sharpe and Dr Ellen I. Garvie (National Institute for Research in Dairying, Shinfield, near Reading) and to Dr P. M. F. Shattock (Department of Microbiology, University of Reading) for generously given advice and helpful criticism, and to Dr G. W. H. Cheeseman (Chemistry Department, Queen Elizabeth College, London) for his assistance during the isolation of lactic acid. Dr Patricia M. Scholes (Bacteriology Department, Queen Elizabeth College, London) has given immense help in the preparation of these papers and we wish to record our appreciation. We would also like to thank Professor M. Rogosa and Dr S. T. Cowan for their helpful criticism of the manuscripts. This work was undertaken while one of us (H.L.G.) was holder of a Postgraduate Research Scholarship awarded by the authorities of Queen Elizabeth College, University of London.

\section{REFERENCES}

DACRE, J. C. (1958). Characteristics of a presumptive pediococcus occurring in New Zealand cheddar cheese. J. Dairy Res. 25, 409.

Felton, E. A. \& Niven, Jr., C. F. (1953). The identity of 'Leuconostoc citrovorum, strain 8081'. J. Bact. 65, 482.

Garvie, E. I. (1959). Reclassification of Leuconostoc mesenteroides P. 60 as a Pediococcus. Nature, Lond. 183, 1411.

GüNTHER, H. L. \& WHITE, H. R. (1961). The cultural and physiological characters of the pediococci. J. gen. Microbiol. 26, 185.

Jensen, E. M. \& Seeley, H. W. (1954). The nutrition and physiology of the genus Pediococcus. J. Bact. 67, 484 .

LANCEFIELD, R. C. (1933). A serological differentiation of human and other groups of hemolytic streptococci. J. exp. Med. 57, 571.

Mickle, H. (1948). Tissue disintegrator. J. R. micr. Soc. 68, 10.

Pederson, C. S. (1949). The genus Pediococcus. Bact. Rev. 13, 225.

Pederson, C. S., Albury, M. N. \& Breed, R. S. (1954). Pediococcus cerevisiae, the beer sarcina. Wallerstein Labs. Communs. 17, 7.

Sharpe, M. E. (1955). A serological classification of lactobacilli. J. gen. Microbiol. 12, 107.

Shatrock, P. M. F. (1949). The streptococci of group D; the serological grouping of Streptococcus bovis and observations on serologically refractory group D strains. J. gen. Microbiol. 3, 80.

Shatrock, P. M. F. \& Hirsch, A. (1947). A liquid medium buffered at pH 9.6 for the differentiation of Streptococcus faecalis from Streptococcus lactis. J. Path. Bact. 59, 496. 